\title{
Time-evolution of Au and Ag nanofluids prepared by direct deposition of gas aggregated nanoparticles into the liquid polymer
}

\author{
Tereza Košutová ${ }^{1}$, Daniil Nikitin², Pavel Pleskunov ${ }^{2}$, Renata Tafiichuk ${ }^{2}$, Andrei Choukourov², Milan Dopita ${ }^{1}$ \\ ${ }^{1}$ Department of Condensed Matter Physics, Faculty of Mathematics and Physics, \\ Charles University, Ke Karlovu 5, 12116, Prague, Czech Republic, \\ ${ }^{2}$ Department of Macromolecular Physics, Faculty of Mathematics and Physics, Charles University, \\ V Holešovičkách 2, Prague, 18000, Czech Republic
}

kosutovat@gmail.com

Nanofluids, i. e. liquids containing dispersed nanoparticles, are gaining increasing interest since the first use of this designation by Choi in 1995 [1]. The primary application for heat transfer as a thermally conductive fluid for cooling is nowadays expanding to sensors, lubricants, magnetic sealing or solar energy collectors. The unique properties of nanofluids arise from the synergy between nanoparticles and the surrounding medium. Our study concerns Ag and Au nanoparticles which belong to plasmonic nanoparticles with the localized particles plasmon resonance (LPPR) in the region of visible light which makes them and their colloidal suspensions attractive for optical applications.

There are numerous preparation methods of nanofluids, among them the very straightforward and solvent-free is magnetron sputtering of metals on the surface of vacuum-compatible liquids (oils, ionic liquids, and polymers). In this method nanoparticles are formed at the vacuum-liquid interface [2]. In our work, the nanoparticle synthesis takes place in the gas phase prior to their landing onto the liquid. Silver and gold nanoparticles were prepared using a magnetron-based gas aggregation cluster source and subsequently deposited into liquid polyethylene glycol (PEG).

The main aim of our study is to determine the stability of Ag and Au nanoparticle dispersions in PEG and to understand the postdeposition processes inside the nanofluids comprising nanoparticles prepared by aggregation from the gas phase. Solutions with different mass concentration of nanoparticles were prepared by controlling the deposition time reaching tens of $\mathrm{mg} / \mathrm{ml}$, a value typical for commercially-available Ag colloidal solutions. To investigate the size distributions and interactions between nanoparticles inside the colloidal suspensions the small angle x-ray scattering (SAXS) was used. We performed SAXS measurements repeatedly during six months to determine the suspension stability. The x-ray diffraction proved the crystalline nature of nanoparticles and also the changes in the amount of material dispersed in the suspension. The optical properties of individual suspensions were analyzed by UV-Vis spectroscopy. TEM and SEM measurements of nanoparticles separated from the suspensions were performed to validate the results obtained by the scattering methods.

Prepared Au nanoparticles have bimodal size distribution with mean sizes $13 \mathrm{~nm}$ and $40 \mathrm{~nm}$ and the corresponding absorption peak associated to the LPPR is observed around $550 \mathrm{~nm}$ in the UV-Vis spectrum. In the case of Ag nanoparticles dispersion, UV-Vis spectroscopy shows the maximum corresponding to the LPPR of individual separated nanoparticles around $410 \mathrm{~nm}$ and another maximum at larger wavelengths corresponding to nanoparticles aggregates for freshly prepared samples. This observation was further confirmed by SAXS, the mean size of single nanoparticles is around $10 \mathrm{~nm}$ and the nanoparticles interact through the hard-sphere interaction. The hard-sphere volume fraction however decreases in time and after two months is not detectable anymore. The resultant suspension exhibited characteristic plasmonic colour in the yellow/orange range and is expected to be stable over extended periods due to constrained mobility of PEG's macromolecular chains.

[1] Choi, S. U. S., \& Eastman, J. A. (1995). American Society of Mechanical Engineers, 231 (March), 99-105.

[2] Wender, H., Gonçalves, R. V., Feil, A. F., Migowski, P., Poletto, F. S., Pohlmann, A. R., Dupont, J., \& Teixeira, S. R. (2011). Journal of Physical Chemistry C, 115(33), 16362-16367.

\section{Keywords: Silver nanofluid; gold nanofluid; time-evolution; gas-aggregation cluster source; SAXS}

This study was financed by the Grant Agency of Charles University (grant 1546119), by the Czech Science Foundation (grant GACR 21-12828S) and by ERDF in the frame of the project NanoCent - Nanomaterials Centre for Advanced Applications (Project No. CZ.02.1.01/0.0/0.0/15_003/0000485). 